\title{
Caracterização da curva da frequência cardíaca durante teste incremental máximo em esteira
}

\author{
Characterization of the heart rate curve during a maximum \\ incremental test on a treadmill
}

\author{
Eduardo Marcel Fernandes Nascimento \\ Adriano Eduardo Lima Silva ${ }^{2}$ \\ Rômulo Cássio Moraes Bertuzzi \\ Maria Augusta Pedutti Dal'Molin Kiss \\ Flavio de Oliveira Pires
}

1 Esporte da Universidade de São Paulo. Escola de Educação Física e Esporte. Laboratório do Desempenho Esportivo. São Paulo, SP. Brasil.

2 Universidade Federal de Alagoas. Faculdade de Nutrição. Grupo de Pesquisa em Ciências do Esporte. Maceió, AL, Brasil.

3 Universidade de São Paulo. Escola de Educação Física e Esporte. Grupo de Estudo em Desempenho Aeróbio. São Paulo, SP. Brasil.

Recebido em 22/10/10 Revisado em 09/01/11 Aprovado em 27/02/11
Resumo - O propósito deste estudo foi analisar o comportamento da frequência cardíaca (FC) versus a carga de trabalho crescente (CTC) em teste de esteira, utilizando três modelos matemáticos (linear, linear com dois segmentos de reta e sigmóide) e verificar qual o melhor modelo que possibilita a identificação de um limiar de FC que pudesse servir de preditor para os limiares ventilatórios $\left(\mathrm{LV}_{1}\right.$ e $\left.\mathrm{LV}_{2}\right)$. Vinte e dois homens realizaram um teste incremental (re-teste: $\mathrm{n}=12$ ), com velocidade inicial de $5,5 \mathrm{~km} \cdot \mathrm{h}^{-1}$ e incrementos de $0,5 \mathrm{~km} \cdot \mathrm{h}^{-1}$ a cada minuto, até a exaustão. Medidas contínuas de FC e trocas gasosas foram convertidas para médias de 5 e 20 segundos. Somatória dos resíduos quadrados e quadrado médio do erro foram usados para verificar o melhor ajuste. A relação FC/CTC foi melhor representada pelo modelo $\mathrm{Lin}_{2}$ no grupo teste e re-teste $(\mathrm{p}<0,05)$. Foi possível identificar um ponto de deflexão de FC, utilizando o modelo $\operatorname{Lin}_{2}$ (limiar de FC) em todos os indivíduos no teste $\left(164 \pm 16,6 \mathrm{bpm} ; 83,6 \% \mathrm{FC}_{\text {MÁX }}\right)$ e no re-teste $\left(162 \pm 20,0 \mathrm{bpm} ; 83,9 \% \mathrm{FC}_{\text {MÁX }}\right)$. O limiar de FC ( $\left.\operatorname{Lin}_{2 \text { PDFC }}\right)$ ocorreu a 9,2 $\pm 1,3 \mathrm{~km} \cdot \mathrm{h}^{-1}\left(67,9 \% \mathrm{Vel}_{\mathrm{MÁX}}\right)$ e foi menor que o $\mathrm{LV}_{2}\left(\mathrm{LV}_{2}=10,6\right.$ $\left.\pm 1,5 \mathrm{~km} \cdot \mathrm{h}^{-1} ; 77,3 \% \mathrm{Vel}_{\mathrm{MÁx}} ; \mathrm{p}<0,05\right)$, mas não diferente de $\mathrm{LV}_{1}\left(8,4 \pm 1,2 \mathrm{~km} \cdot \mathrm{h}^{-1} ; 61,6 \%\right.$ $\left.\mathrm{Vel}_{\text {MÁx }} ; \mathrm{p}>0,05\right)$. Durante teste incremental em esteira, a relação FC/CTC parece ser bem descrita por uma função linear com 2 segmentos de reta, a qual permite a determinação de um limiar de FC que se aproxima do $\mathrm{LV}_{1}$.

Palavras-chave: Ajuste sigmóide de Boltzmann; Ergometria; Teste de esteira rolante; Esforço físico; Frequência cardíaca

Abstract - The objective of this study was to analyze the heart rate (HR) profile plotted against incremental workloads (IWL) during a treadmill test using three mathematical models [linear, linear with 2 segments ( $\operatorname{Lin}_{2}$ ), and sigmoidall, and to determine the best model for the identifcation of the HR threshold that could be used as a predictor of ventilatory thresholds (VT, and $\mathrm{VT}_{2}$ ). Twenty-two men underwent a treadmill incremental test (retest group: $n=12$ ) at an initial speed of $5.5 \mathrm{~km} \cdot \mathrm{h}^{-1}$, with increments of $0.5 \mathrm{~km} \cdot \mathrm{h}^{-1}$ at 1 -min intervals until exhaustion. HR and gas exchange were continuously measured and subsequently converted to 5-s and 20-s averages, respectively. The best model was chosen based on residual sum of squares and mean square error. The HR/IWL ratio was better fitted with the Lin, model in the test and retest groups $(p<0.05)$.

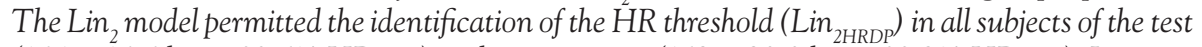
$\left(164 \pm 16.6 \mathrm{bpm} ; 83.6 \% \mathrm{HR}_{\mathrm{MAX}}\right)$ and retest groups $\left(162 \pm 20.0 \mathrm{bpm} ; 83.9 \% \mathrm{HR}_{\mathrm{MAX}}\right) . \operatorname{Lin}_{2 \mathrm{HRDP}}$ $\left(9.2 \pm 1.3 \mathrm{~km} \cdot \mathrm{h}^{-1} ; 67.9 \%\right.$ speed $\left._{\text {MAX }}\right)$ was lower than VT $\left(10.6 \pm 1.5 \mathrm{~km} \cdot \mathrm{h}^{-1}, 77.3 \%\right.$ speed $_{\text {MAX }}$; $p<0.05)$, but did not differ from VT $18.4 \pm 1.2 \mathrm{~km} \cdot \mathrm{h}^{-1}, 61.6 \%$ speed $\left._{\text {MAx }} ; p>0.05\right)$. During a treadmill incremental test, the HR/IWL ratio seems to be better fitted with a Lin 2 model, which permits to determine the HR threshold that coincides with $\mathrm{VT}_{1}$.

Key words: Boltzmann sigmoidal model; Ergometry; Exercise test; Physical exertion; Heart rate. 


\section{INTRODUÇÃO}

Modelos lineares para descrição da relação frequência cardíaca (FC) e carga de trabalho crescente (CTC) têm sido formulados desde o século passado ${ }^{1}$. Mesmo diante de trabalhos que demonstraram as limitações do modelo linear ${ }^{2,3}$, sua fácil aplicação e a possibilidade da predição da capacidade máxima aeróbia parecem ter sido os principais fatores que explicam a preferência desta função quando da descrição da relação FC/CTC ${ }^{4}$.

Evidências iniciais das limitações do modelo linear foram observadas por Conconi et al. ${ }^{2}$, analisando a parte superior da curva da FC em teste incremental. Foi identificado um aumento não linear da relação FC/CTC, com a presença de um ponto de deflexão da FC (PDFC), posteriormente, associado ao segundo limiar de lactato 5 . Por apresentar um baixo custo e não requerer técnicas invasivas ${ }^{6,7}$, o PDFC, frequentemente identificado de forma visual, passou a ser amplamente utilizado por técnicos e treinadores para estimativa do segundo limiar de transição metabólica ${ }^{8}$. Evidências também foram descritas quando analisada a porção inferior da curva FC/CTC 9,10. Pfeiffer \& Steyer ${ }^{9}$, e Crowhurst et al. ${ }^{10}$ sugeriram a existência de um ponto de mudança na parte inferior da curva $\mathrm{FC} /$ CTC, o ponto de inflexão da FC.

Quando confrontados os resultados dos trabalhos que descreveram a relação FC/CTC em sua parte inferior ${ }^{9,10}$, com os da parte superior ${ }^{2}$, fica a sugestão de que essa relação possa ser descrita por uma função sigmóide, em forma de "S". De fato, Lima ${ }^{3}$ sugeriu a utilização do ajuste sigmóide de Boltzmann para caracterizar o comportamento da FC durante teste progressivo máximo em cicloergômetro. Outros estudos têm comparado a aplicabilidade do modelo linear em relação ao sigmóide $^{11,12}$, demonstrando que o ajuste sigmóide pode caracterizar melhor a relação FC/CTC.

No entanto, todos estes estudos utilizaram cicloergômetro de membros inferiores para a comparação dos modelos linear e sigmóide, restando dúvidas se um comportamento sigmóide também seria a melhor caracterização desta relação em outros tipos de ergômetros. Diferentemente da bicicleta, há um ponto claro de modificação da eficiência metabólica ${ }^{13}$, durante testes incrementais em esteira ${ }^{14}$. Após as fases inicias de um teste em esteira, existe um momento de transição entre a caminhada e a corrida, durante o qual há uma diminuição da eficiência mecânica, resultando em aumento da demanda energética envolvida na tarefa ${ }^{15}$. Desta forma, seria razoável supor que esta perda de eficiência mecânica na transição caminhada-corrida possa gerar uma maior taxa de aumento da $\mathrm{FC}$ entre as partes inicial e central da curva FC/CTC, mudando o padrão sigmóide da relação FC/CTC observada em cicloergômetro.

Um ponto importante a ser considerado é o caráter aplicado da curva da FC. O PDFC tem se mostrado útil para a determinação do nível de aptidão aeróbia e prescrição de treinamento ${ }^{4}$, pois permite a identificação de um limiar de transição metabólica ${ }^{5}$. Primeiramente, contudo, ele é baseado num comportamento não linear da curva FC/CTC ${ }^{2}$. Em adição, estudos mostram que o PDFC pode variar de acordo com o método empregado. Por exemplo, como o método visual apresenta limitações ${ }^{16}$, estudos propuseram modelos matemáticos para melhorar a acurácia da sua identificação ${ }^{17}$. Portanto, além de verificar qual função descreve melhor a relação FC/CTC em esteira, é necessário saber qual o melhor ajuste que possibilitaria a identificação de um limiar de transição metabólica, com alguma dependência do método usado.

Desta forma, o objetivo deste estudo foi o de analisar o comportamento da relação FC/CTC durante teste máximo em esteira rolante, utilizando três ajustes matemáticos (linear, sigmóide e linear com dois segmentos de reta) para a identificação do limiar de transição metabólica.

\section{PROCEDIMENTOS METODOLÓGICOS}

\section{Sujeitos}

Vinte e dois homens saudáveis $(25,0 \pm 2,9$ anos; $175,3 \pm 6 \mathrm{~cm} ; 74,9 \pm 8,6 \mathrm{~kg}$ ), sem qualquer restrição clínica, fizeram parte do estudo. Os protocolos de intervenção foram aprovados pelo Comitê de Ética em Pesquisa da Universidade de São Paulo, protocolo $n^{\circ} 75$ (aprovado no CEP EEFE em 26/11/2004). Os sujeitos foram orientados a evitar atividades físicas extenuantes 24 horas antes dos testes, a consumir refeições leves duas horas antes e a não ingerir bebidas com conteúdo de cafeína ou álcool.

\section{Teste incremental máximo em esteira}

$O$ teste incremental foi realizado em esteira (Sensormedics ${ }^{\circledR}$ Vmax 2000), com um protocolo adaptado de Conconi ${ }^{2}$. Após 3 minutos em 5,5 km.h.' a velocidade (Vel.) foi aumentada em $0,5 \mathrm{~km} \cdot \mathrm{h}^{-1} \mathrm{a}$ cada minuto, até a exaustão. A esteira foi mantida com $1 \%$ de inclinação durante todo o teste. Todo o 
procedimento experimental foi repetido em 12 dos 22 sujeitos da amostra (grupo reteste $=$ GR; 24,75 $\pm 3,7$ anos; $176,33 \pm 6,42 \mathrm{~cm} ; 74,77 \pm 9,9 \mathrm{~kg})$. A FC foi mensurada durante o teste (Polar Vantage NV), e a média dos 5 segundos finais de cada estágio foi registrada. As trocas gasosas foram registradas continuamente, através de um analisador de gases de circuito aberto (Sensormedics®, Yorba Linda, Califórnia, USA), calibrado antes de cada teste, seguindo as instruções do fabricante. Uma seringa de três litros e cilindros com gases de composição conhecida foram utilizados para calibrar o sensor de fluxo e os analisadores de $\mathrm{O}_{2}$ e $\mathrm{CO}_{2}$.

\section{Ajuste das curvas}

Os dados da FC foram plotados em função do tempo, para construção dos seguintes modelos matemáticos:

Linear (Lin): $y=a+b(x)$

onde, y é o valor predito da FC, a é o intercepto, $b$ representa a inclinação da reta e $x$ é a velocidade (Figura 1A);

Segmentada de 2 retas $\left(\operatorname{Lin}_{2}\right)$ : descreve o comportamento em forma de uma reta segmentada em duas fases (Figura 1B), com um ponto de quebra na linearidade (intersecção):

$y=a+b(x)\left(1^{o}\right.$ segmento $)$

$y=a+\alpha+b_{2}(x)\left(2^{\circ}\right.$ segmento $)$

onde, y é o valor predito da FC, a é o intercepto de cada segmento, $\alpha$ é o ponto tangencial entre os segmentos 1 e 2 , empregado para obter o intercepto do $2^{\circ}$ segmento, $b_{2}$ representa a inclinação de cada segmento, e $x$ é a velocidade. Alguns critérios foram considerados nos cálculos deste modelo: 1) o ponto de intersecção entre as duas retas foi obtido, testando-se todas as possíveis combinações com um mínimo de três pontos para cada segmento; 2) o intercepto que gerou o ajuste com maior valor de $r^{2}$ e menor erro padrão de estimativa foi adotado.

Sigmóide (Sig): descreve o comportamento em forma de "S" (Figura 1C), segundo a seguinte equação (c):

$y=\left(A_{1}-A_{2} / 1+e^{(x-x o) / d x}\right)+A_{2}$

onde o y é o valor predito de FC, $x$ é o valor da carga de trabalho $\left(\mathrm{km} \cdot \mathrm{h}^{-1}\right), A_{1}$ e $A_{2}$ correspondem à linha de base $\mathrm{e}$ superior de FC, respectivamente, $\mathrm{x}_{0}$ é o valor central da curva obtido pela primeira derivada da função, e $d x$ é a constante de crescimento da curva.

\section{Análise residual}

A análise da qualidade dos ajustes das curvas foi obtida pela somatória dos resíduos quadrados (SRQ) e o quadrado médio do erro (QME) dos resíduos. A SQR foi obtida pela soma do quadrado da diferença entre os valores medidos e preditos da FC, o QME foi obtido pela SRQ corrigida pela diferença entre o número de pontos contidos em cada curva individual e o número de parâmetros de cada função matemática ${ }^{18,19}$. Quando necessário, adotou-se o princípio da parcimônia para estabelecer o melhor ajuste. Este princípio prevê que o ajuste mais simples seja adotado, quando da ausência de diferença significante nos resíduos gerados pelos ajustes.

\section{Identificação dos limiares de FC}

Após determinar a melhor função matemática que descreveu o comportamento da relação FC/ CTC, três diferentes métodos foram empregados nos valores estimados de FC para a determinação do PDFC (Figura 1): 1) Visual (Visual ${ }_{\text {PDFC }}$ ): identificado visualmente (mediana de três avaliadores), no último ponto antes de ocorrer aumento abrupto na FC; 2) Distância máxima (Dmáx ${ }_{\mathrm{PDFC}}$ ): a maior distância encontrada entre os valores de FC estimados pela melhor função matemática e uma equação linear obtida a partir do primeiro valor de $\mathrm{FC} \geq 140$ bpm e o último valor da curva ${ }^{17}$; 3) Linear com dois segmentos de reta $\left(\operatorname{Lin}_{2 \mathrm{PDFC}}\right)$ : identificado na intersecção entre os dois segmentos do ajuste Lin $_{2}$. Todos os valores referentes ao PDFC identificado pelos diferentes métodos foram expressos em valores absolutos [Vel (km.h-1), FC (bpm) e VO $\left(\mathrm{ml} \cdot \mathrm{kg}^{-1}\right.$. $\left.\left.\mathrm{min}^{-1}\right)\right]$ e relativos aos valores máximos $\left(\% \mathrm{Vel}_{\mathrm{MÁX}}\right.$ \%FC $\mathrm{FÁX}_{\text {e }}$ \% VO $\left.{ }_{2 \mathrm{PICO}}\right)$.

\section{Identificação dos limiares ventilatórios}

Análises dos equivalentes ventilatórios de $\mathrm{O}_{2}$ $\left(\mathrm{VE} / \mathrm{VO}_{2}\right)$ e $\mathrm{CO}_{2}\left(\mathrm{VE} / \mathrm{VCO}_{2}\right)$ identificaram o primeiro $\left(\mathrm{LV}_{1}\right)$ e segundo limiar ventilatório $\left(\mathrm{LV}_{2}\right)^{20}$. Após conversão dos dados brutos para médias de 20 segundos, a inspeção visual (mediana de três avaliadores) das curvas de $\mathrm{VE} / \mathrm{VO}_{2}$ e $\mathrm{VE} / \mathrm{VCO}_{2}$ foi utilizada para identificar $L V_{1}$ e $L V_{2}$, respectivamente. Quando os dados dos VE/ $\mathrm{VO}_{2}$ e VE/VCO não apresentaram nitidez para a identificação dos limiares ventilatórios, as frações expiradas de oxigênio $\left(\mathrm{LV}_{1}\right)$ e dióxido de carbono $\left(\mathrm{LV}_{2}\right)$ foram utilizadas. Valores absolutos [Vel $\left(\mathrm{km} . \mathrm{h}^{-1}\right), \mathrm{FC}$ $(\mathrm{bpm})$ e $\left.\mathrm{VO}_{2}\left(\mathrm{ml} \mathrm{kg}^{-1} \cdot \mathrm{min}^{-1}\right)\right]$ e relativos $\left(\% \mathrm{Vel}_{\mathrm{MÁX}}\right.$ $\% \mathrm{FC}_{\text {MÁX }}$ e $\% \mathrm{VO}_{2 \mathrm{PICO}}$ ) foram utilizados para expressar $\mathrm{LV}_{1}$ e $\mathrm{LV}_{2}$. $\mathrm{O} \mathrm{VO}_{2}$ de pico $\left(\mathrm{VO}_{2 \mathrm{PICO}}\right)$ foi estabelecido pela média dos três maiores valores alcançados. 

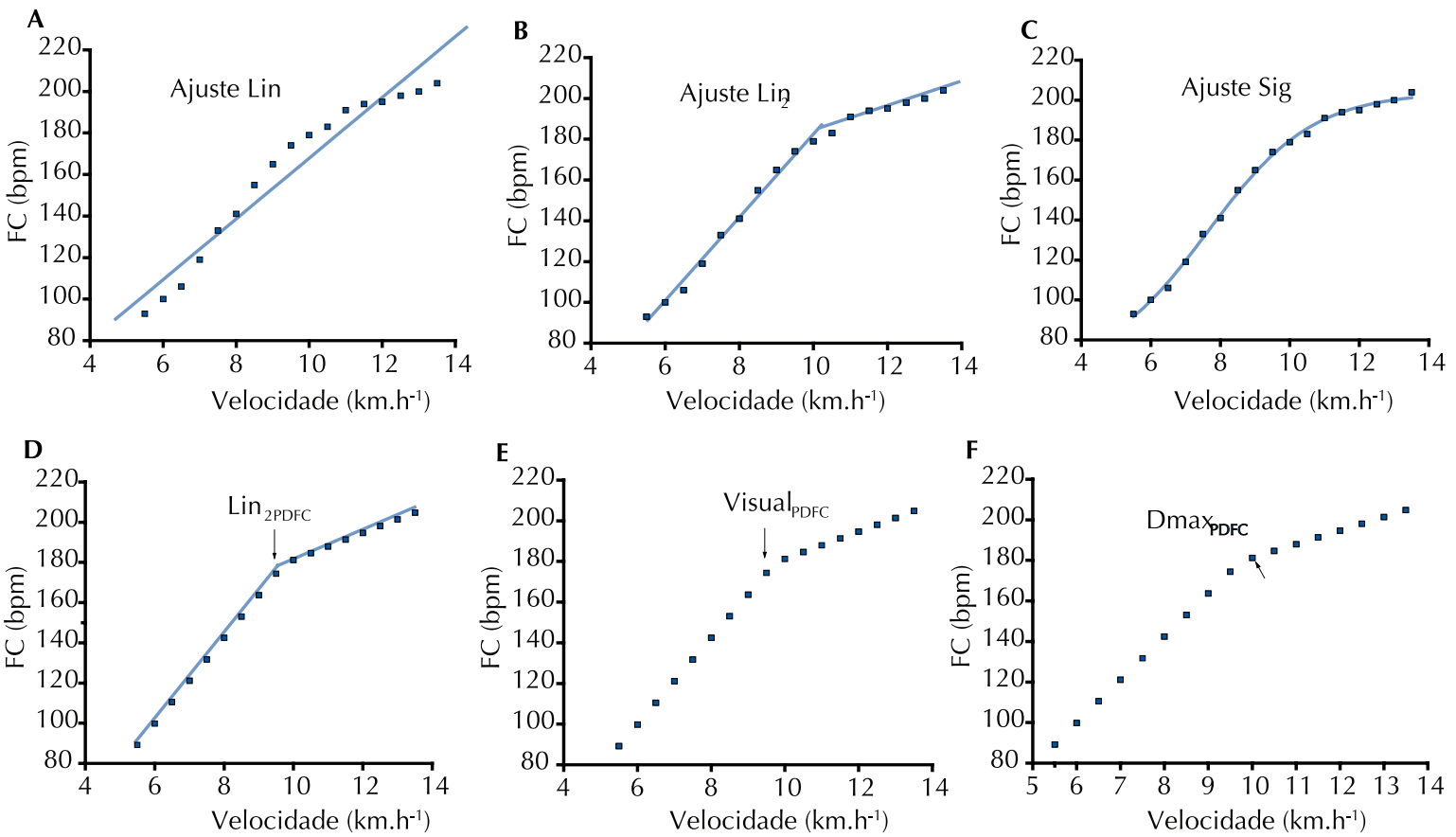

Figura 1. Representação gráfica da caracterização da FC [A) ajuste linear (Lin); B) ajuste segmentado duas restas (Lin ${ }_{2}$ ); e C) ajuste sigmóide de Boltzmann (Sig)] e da identificação do PDFC através dos modelos $\operatorname{Lin}_{2 \mathrm{PDFC}}(\mathrm{D})$, $_{\text {, }}$ isual $_{\mathrm{PDFC}}(\mathrm{E}) \mathrm{e}^{\mathrm{Dmax}} \mathrm{DmFC}_{\mathrm{P}}(\mathrm{F})$.

\section{Análise estatística}

Os dados são expressos em média e desvio padrão $( \pm \mathrm{dp})$. A distribuição dos dados foi verificada pelo teste de Shapiro-Wilk. ANOVA de Friedman, acompanhada por post hoc de Wilcoxon, foi utilizada para verificar as diferenças na SRQ e QME entre os três modelos matemáticos. $O$ coeficiente de correlação de Pearson foi utilizado para expressar o nível de correlação entre os valores medidos e preditos por cada ajuste matemático. ANOVA One-way e post hoc de Bonferroni foram utilizados para verificar as diferenças entre o PDFC identificado pelos diferentes métodos e os limiares ventilatórios. O coeficiente de correlação intraclasse (CCI) foi utilizado para testar o nível de reprodutibilidade das variáveis obtidas para o GT e GR. Um nível de significância de 5\% foi adotado $(p<0,05)$.

\section{RESULTADOS}

Os valores alcançados de $\mathrm{FC}_{\mathrm{MÁX}}, \mathrm{VE}_{\mathrm{MÁX}}, \mathrm{Vel}_{\mathrm{MÁX}}$, $\mathrm{VO}_{2 \mathrm{PICO}}$ absoluto e $\mathrm{VO}_{2 \mathrm{PICO}}$ relativo foram $196 \pm 8,5$ bpm, 126,7 \pm 17,9 L.min min $^{-1}$ 13,6 \pm 1,4 km.h ${ }^{-1}, 3,74 \pm$ 0,6 L. $\mathrm{min}^{-1}$ e $49,9 \pm 6,3 \mathrm{ml} \cdot \mathrm{kg}^{-1} \cdot \mathrm{min}^{-1}$, respectivamente.

\section{Ajuste das curvas}

A figura 2 representa a plotagem dos valores da SRQ para os diferentes tipos de ajuste, com as suas respectivas linhas de tendência dos resíduos.

No GT, o ajuste Lin apresentou valores de SRQ maiores $(\mathrm{p}<0,05)$ do que os ajustes Sig e $\mathrm{Lin}_{2}$ (Tabela 1). No entanto, não houve diferença nos valores dos SQR e QME entre os ajustes Lin e Sig ( $p>0,05)$. Resultados idênticos foram encontrados no GR, já que SRQ e QME também foram maiores
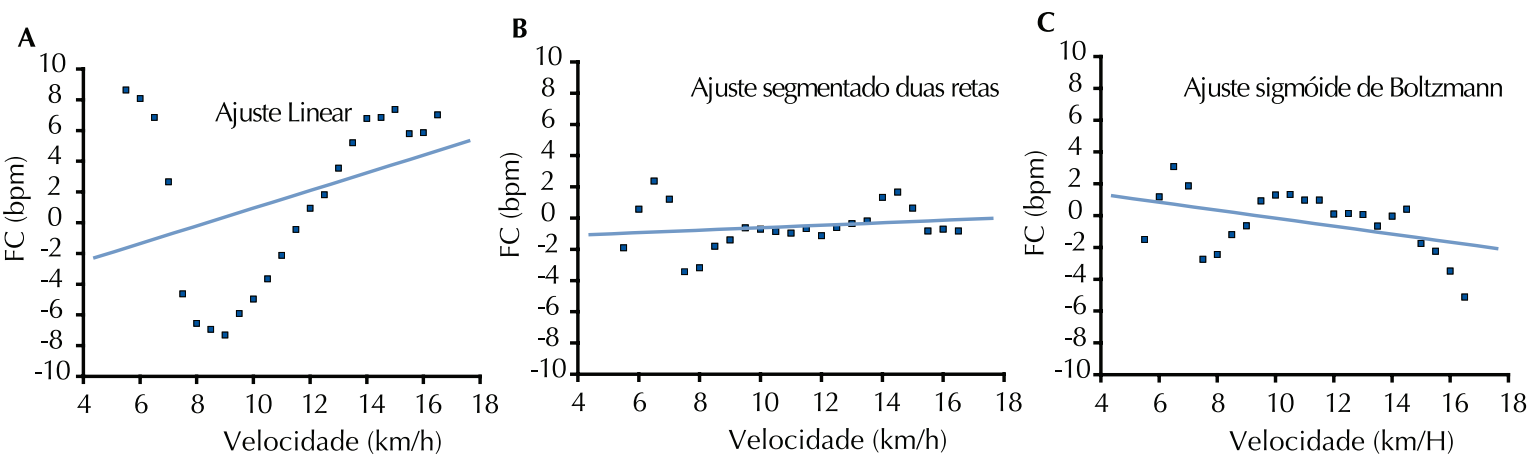

Figura 2. Representação gráfica dos valores médios referentes aos SRQ dos diferentes ajustes matemáticos obtidos durante o teste: $\operatorname{Lin}(\mathrm{A}) \operatorname{Lin}_{2}(\mathrm{~B})$ e Sig (C). 
Tabela 1. Média e desvio padrão dos parâmetros SRQ e QME dos ajustes matemáticos.

\begin{tabular}{|c|c|c|c|c|c|c|}
\hline & & GT & & & GR & \\
\hline & Lin & $\operatorname{Lin} 2$ & Sig & Lin & $\operatorname{Lin} 2$ & Sig \\
\hline SRQ & $799,1 \pm 446,9$ & $199,0 \pm 192,2 *$ & $131,1 \pm 90,7^{*}$ & $831,2 \pm 475,8$ & $128,9 \pm 93,1 *$ & $121,0 \pm 74,3^{*}$ \\
\hline QME & $52,4 \pm 23,0$ & $14,4 \pm 20,5^{*}$ & $9,7 \pm 6,1^{*}$ & $53,1 \pm 5,2$ & $8,2 \pm 5,9^{*}$ & $8,8 \pm 5,2 *$ \\
\hline
\end{tabular}

$\overline{\mathrm{GT}}=$ grupo teste; $\mathrm{GR}=$ grupo reteste. ${ }^{*}$ diferença significante em relação ao ajuste $\mathrm{Lin}(\mathrm{p}<0,05)$.

no ajuste Lin. Altos níveis de reprodutibilidade foram encontrados para os ajustes Lin (SRQ: CCI= 0,84; $<<0,01$; QME: $C C I=0,85 ; p<0,01$ ), $\operatorname{Lin}_{2}$ (SRQ: $\mathrm{CCI}=0,49 ; \mathrm{p}=0,13$; QME: $\mathrm{CCI}=0,55 ; \mathrm{p}=0,09)$ e Sig (SRQ: CCI=0,77; p<0,01; QME: $\mathrm{CCI}=0,71$; $\mathrm{p}<0,05)$.

\section{PDFC e limiares ventilatórios}

O PDFC identificado pelos diferentes métodos e os limiares ventilatórios são apresentados na tabela 2. $\mathrm{O}$ modelo $\mathrm{Lin}_{2}$ foi adotado como o ajuste que melhor descreveu a curva FC/CTC. Os valores de FC ajustados por esta função possibilitaram a identificação de um limiar pelos métodos $\operatorname{Lin}_{2 \mathrm{PDFC}}$

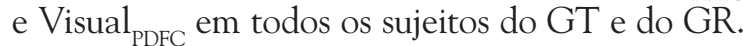
No entanto, não foi possível identificar o PDFC pelo método Dmáx (Dmáx ${ }_{\mathrm{PDFC}}$ ) em dois sujeitos do GT e em um sujeito do GR.

Não foi observada diferença no PDFC identificado pelos diferentes métodos, expressos em valores absolutos ou relativos $(p<0,05)$. Significantes níveis de CCI foram observados para o método $\operatorname{Lin}_{2 \mathrm{PDFC}}(\mathrm{CCI}=0,84 ; \mathrm{p}<0,01)$, Visual $_{\mathrm{PDFC}}(\mathrm{CCI}=$ $0,87 ; p<0,01)$, mas não para o método Dmáx $\mathrm{PDFC}$ $(\mathrm{CCI}=0,00 ; \mathrm{p}>0,05)$.

Quando comparados aos limiares ventilatórios, o PDFC foi menor que o $\mathrm{LV}_{2}$, quando expressos em função da velocidade absoluta ou em \% $\operatorname{Vel}_{\text {MÁx }}$ ( $\mathrm{p}<$ $0,05)$. Contudo, quando os limiares foram expressos em valores absolutos de $\mathrm{FC}$ e em valores relativos à $\% \mathrm{FC}_{\mathrm{MAX}}$, $\mathrm{PDFC}$ foi maior que o $\mathrm{LV}_{1}(\mathrm{p}<0,05)$. Estes resultados estão apresentados na tabela 2.

A figura 3 mostra a representação gráfica da demanda metabólica expressa pelos valores individuais da relação $\mathrm{VO}_{\text {2relativo }} / \mathrm{Vel}$.

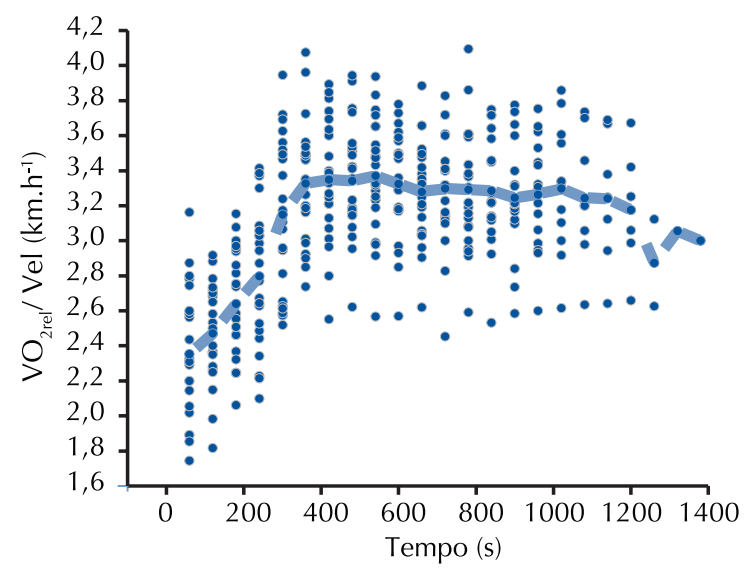

Figura 3. Representação gráfica da demanda metabólica expressa pelos valores individuais da relação $\mathrm{VO}_{\text {2relativo }} / \mathrm{Vel}$. referentes ao 22 sujeitos e a linha média (linha escura).

\section{DISCUSSÃO}

O presente estudo testou a utilização de três ajustes matemáticos para caracterizar a relação FC/CTC em teste incremental em esteira. Os principais resultados corroboram estudos anteriores conduzidos em cicloergômetro de membros inferiores ${ }^{11,3}$, demonstrando que o modelo linear não descreve bem o comportamento da FC também durante teste em esteira. Por outro lado, diferentemente daqueles estudos anteriores, a presente investigação incluiu um terceiro ajuste, o ajuste segmentado de 2 retas. A hipótese de que o ajuste sigmóide pudesse descrever melhor o comportamento da relação FC/CTC não foi confirmada, uma vez que o $\operatorname{Lin}_{2}$ foi aceito como o melhor ajuste, baseado no princípio da parcimônia. Outro resultado importante foi a possibilidade de identificação do PDFC em esteira a partir dos valores de FC preditos pelo ajuste $\operatorname{Lin}_{2 \mathrm{PDFC}}$.

Tabela 2. Valores médios dos vinte e dois sujeitos, referentes aos pontos de identificação dos diferentes métodos.

\begin{tabular}{lcccccc}
\hline & $(\mathrm{bpm})$ & $(\%$ máx. $)$ & $\left(\mathrm{ml}^{\mathrm{kg}} \cdot \mathrm{min}^{-1}\right)$ & $\left(\%_{\mathrm{PICO}}\right)$ & $\left(\mathrm{km} \cdot \mathrm{h}^{-1}\right)$ & $(\%$ máx. $)$ \\
\hline $\mathrm{LV}_{1}$ & $147 \pm 16,9$ & $75,3 \pm 8,1$ & $26,9 \pm 5,2^{\ddagger}$ & $53,8 \pm 7,8$ & $8,4 \pm 10,6^{\ddagger}$ & $61,6 \pm 6,6^{\ddagger}$ \\
$\mathrm{LV}_{2}$ & $172 \pm 12,4^{* *}$ & $87,9 \pm 4,5^{* *}$ & $35,2 \pm 5,1$ & $70,6 \pm 4,5^{* *}$ & $10,6 \pm 1,5$ & $77,4 \pm 5,3$ \\
$\mathrm{Lin}_{2}$ & $164 \pm 16,6^{*}$ & $83,6 \pm 7,7^{* *}$ & $31,1 \pm 4,3$ & $63,1 \pm 10,7^{*}$ & $9,2 \pm 1,3^{\ddagger}$ & $68,0 \pm 10,8^{+}$ \\
Visual & $164 \pm 16,7^{*}$ & $83,6 \pm 7,6^{* *}$ & $31,0 \pm 4,4$ & $63,0 \pm 11,2^{*}$ & $9,2 \pm 1,3^{\ddagger}$ & $68,1 \pm 10,8^{+}$ \\
Dmáx & $164 \pm 19,9^{*}$ & $83,0 \pm 10,4^{*}$ & $30,6 \pm 5,8^{+}$ & $62,0 \pm 12,5$ & $9,2 \pm 1,3^{\neq}$ & $67,9 \pm 12,2^{+}$ \\
\hline
\end{tabular}

$*(p<0,05), * *(p<0,01)$ diferença estatisticamente significativa em relação ao métvodo LV1,

$+(p<0,05), \neq(p<0,01)$ diferença estatisticamente significativa em relação ao método LV2. 


\section{Ajustes das curvas da FC}

Não foram encontrados estudos que descrevessem o comportamento da FC durante teste progressivo máximo em esteira, com o emprego de três diferentes modelos matemáticos. Estudos anteriores propuseram um padrão sigmóide da FC durante teste incremental em cicloergômetro, após comparação com o ajuste linear ${ }^{11,3,12}$. No presente estudo, o ajuste sigmóide também se mostrou melhor do que o ajuste Lin, quando analisados o SRQ ou o QME.

Como o ajuste Lin, foi adotado como o melhor ajuste, baseado no princípio da parcimônia (mais simples), pressupõe que o ajuste Sig não conseguiu melhorar a descrição da relação FC/CTC em teste em esteira, mesmo com a inclusão de mais parâmetros matemáticos. Isto poderia estar relacionado à perda da eficiência mecânica durante a fase de transição entre caminhada e corrida, em esteira ${ }^{14,15}$. De fato, análise adicional dos dados do presente estudo pode reforçar este argumento, uma vez que a relação $\mathrm{VO}_{2} /$ carga de trabalho (expresso em $\mathrm{VO}_{2 \text { relativo }} / \mathrm{km}^{-1} \mathrm{~h}^{-1}$ ), nas velocidades entre 6 e $8 \mathrm{~km} \cdot \mathrm{h}^{-1}$, foi significantemente maior do que nas velocidades maiores (Figura 3). Entretanto, como o ajuste $\operatorname{Lin}_{2}$ não foi investigado nos estudos anteriores ${ }^{3,11,12}$, não é possível saber se o padrão encontrado na relação FC/CTC em esteira poderia ser encontrado também em bicicleta.

Análise da dispersão residual mostrou que o ajuste Lin superestima os valores de FC nos momentos iniciais e finais do teste, com tendência à subestimação dos valores da parte central da curva (Figura 2). Esses valores se distanciaram razoavelmente dos valores medidos $\left(4,1 \%\right.$ da $\left.\mathrm{FC}_{\mathrm{MÁX}}\right)$. O ajuste $\operatorname{Lin}_{2}$ apresentou um padrão de dispersão mais aleatório, principalmente na grande parte central da curva, com menor dispersão $\left(1,8 \%\right.$ da $\left.\mathrm{FC}_{\mathrm{MÁX}}\right)$. $\mathrm{O}$ ajuste Sig tendeu a um padrão de dispersão similar ao $\operatorname{Lin}_{2}$, porém com dispersão maior para os momentos iniciais e finais do teste. Estes resultados poderiam ser interpretados como uma evidência de que o comportamento da FC durante teste incremental em esteira parece ser bem caracterizado pelos ajustes $\mathrm{Lin}_{2}$ e Sig, sendo que o primeiro, mais simples, utiliza menos parâmetros para a estimativa dos valores de FC.

\section{Métodos de identificação dos limiares}

Os resultados do presente estudo mostram que a relação FC/CTC apresenta um aumento bastante próximo de uma função linear nas velocidades compreendidas entre o início e $~ 70 \%$ da velocidade máxima atingida. Deste ponto em diante, há uma redução na taxa de elevação da FC, o que possibilita a identificação de um PDFC. Como ressaltado anteriormente, este comportamento poderia estar associado à perda de eficiência mecânica em esteira, nas velocidades intermediárias. De fato, o PDFC identificado pelo $\operatorname{Lin}_{2 \mathrm{PDFC}}$ se situou em torno de $9,2 \mathrm{~km} \cdot \mathrm{h}^{-1}$, acima das velocidades normalmente envolvidas na transição entre a caminhada e a corrida $\left(6,5-7,5 \mathrm{~km} \cdot \mathrm{h}^{-1}\right)^{14}$.

O ajuste $\mathrm{Lin}_{2}$ pôde fornecer um PDFC em todos os sujeitos do GT e do GR, quando identificado matematicamente na intersecção dos dois segmentos de reta $\left(\operatorname{Lin}_{2 \mathrm{PDFC}}\right)$. Este ajuste foi obtido testando todas as possíveis intersecções entre as duas retas, tendo como critério um número mínimo de três pontos para cada segmento. Além disso, toda a amplitude da curva FC/CTC foi analisada. Este detalhe metodológico difere do empregado por outros estudos, os quais descartavam os valores iniciais de $\mathrm{FC}$, inferiores à $140 \mathrm{bpm}^{17}$.

De outro lado, também foi utilizado um método que empregava apenas valores de $\mathrm{FC} \geq 140 \mathrm{bpm}$, para a identificação do PDFC (Dmáx). Diferentemente do ajuste polinomial de terceira ordem utilizado por Kara et al. ${ }^{17}$, usamos os valores de FC obtidos pelo melhor ajuste matemático, o $\operatorname{Lin}_{2}$. Os resultados do PDFC obtidos por este método, no presente estudo, estão em torno de $83 \% \mathrm{FC}_{\mathrm{MÁX}}$, abaixo dos valores em torno de $90 \% \mathrm{FC}_{\mathrm{MAx}}$ encontrados por Kara et al. ${ }^{17}$ e Ribeiro et al. ${ }^{5}$. Em adição, estes estudos encontraram um PDFC coincidente com o segundo limiar de transição metabólica, enquanto o PDFC encontrado no presente estudo foi coincidente com o $\mathrm{LV}_{1}$ (expresso em $\mathrm{km} \cdot \mathrm{h}^{-1}$ ). Juntos, estes resultados podem sugerir que a coincidência do PDFC com a primeira ou a segunda zona de transição metabólica pode depender do ajuste empregado antes da aplicação do método.

Os resultados do presente estudo revelaram não haver diferenças significantes entre os limiares identificados pelos métodos $\operatorname{Lin}_{2 \mathrm{PDFC}}$, Dmáx $\mathrm{PDFC}_{\mathrm{P}} \mathrm{e}$ Visual $_{\text {PDFC }}$. Em adição, com a exceção do método Dmáx $_{\mathrm{PDFC}}$, todos apresentaram alto nível de reprodutibilidade no re-teste. Levando-se em consideração que estes métodos não foram diferentes do $\mathrm{LV}_{1}$, torna-se sugestivo que eles sirvam de aproximação da intensidade da primeira zona de transição metabólica, em testes máximos conduzidos em esteira. No entanto, devemos ressaltar que quando estes limiares de FC foram expressos em valores percentuais ao $\mathrm{VO}_{2 \mathrm{PICO}}$, eles se distanciaram do $\mathrm{LV}_{1}$. De certa forma, isto poderia condicionar a utilização destes limiares de FC como aproximação de $\mathrm{LV}_{1}$ em esteira, apenas quando expressos em função da velocidade. 


\section{CONCLUSÃO}

A FC parece ser melhor descrita por um ajuste $\mathrm{Lin}_{2}$, quando comparada aos ajustes linear e sigmóide de Boltzmann, durante teste incremental máximo em esteira. $O$ ponto de intersecção entre as duas retas deste ajuste $\left(\operatorname{Lin}_{\text {2PDFC }}\right)$ parece ser uma razoável aproximação do primeiro limiar de transição metabólica, identificado pelo $\mathrm{LV}_{1}$. Futuros estudos devem ser desenhados para responder se a caracterização da relação FC/CTC encontrada em esteira está, de fato, ligada à queda da eficiência mecânica na transição entre caminhada e corrida.

\section{REFERÊNCIAS BIBLIOGRÁFICAS}

1. Wahlund H. Determination of physical capacity. Acta Physiol Scand 1948;215(suppl. 1):I-78.

2. Conconi F, Ferrari M, Ziglio PG, Droghetti P, Codeca $\mathrm{L}$, Determination of the anaerobic threshold by a noninvasive field test in runners. J Appl Physiol, Bethesda 1982;52(4):869-73.

3. Lima JRP. Freqüência cardíaca em cargas crescentes de trabalho: Ajuste sigmóide, ponte de inflexão e limiar de variabilidade da freqüência cardíaca. [Tese de Doutorado - Programa de Pós-Graduação em Biodinâmica do Movimento Humano]. São Paulo (SP): Escola de Educação Física e Esporte da Universidade de São Paulo; 1997.

4. Achten J, Jeukendrup AE. Heart Rate Monitoring: Applications and Limitations. Sports Med 2003;33(7):517-38.

5. Ribeiro JP, Fielding RA, Hughes V, Black A, Bochese MA, Knuttgen HG. Heart rate break point may coincide with the anaerobic threshold and not the aerobic threshold. Int J Sports Med 1985;6(4):220-24.

6. Bodner ME, Rhodes EC. A review of the concept of the heart rate deflection point. Sports Med 2000;30(1):31-46.

7. Bodner ME, Rhodes EC, Martin AD, Coutts KD. The relationship of the heart rate deflection point to the ventilatory threshold in trained cyclists. J Strength Cond Res 2002;16(4):573-580.

8. Petit MA, Nelson CM, Rhodes EC. Comparison of a mathematical model to predict $10-\mathrm{Km}$ performance from the Conconi test and ventilatory threshold measurements. Can J Appl Physiol 1997;22(6):562-72.

9. Pfeiffer KP, Steyer GE. Determination of physical working capacity in children using three different regression models. Int J Sports Med 1984;5(2):83-8.

10. Crowhurst ME, Morrow JR, Pivarnik JM, Bricker JT. Determination of the aerobic benefit of selected physical education activities. Res Q Exerc Sport 1993;64(2):223-6.
11. Costa VP, Lima JRP, De-Oliveira FR. Identificação de Limiares metabólicos em curvas de frequência cardíaca ajustadas. Rev Bras Educ Fís Esp 2007;21(3):219-27.

12. Piovezana PS, De-Oliveira FR. Reprodutibilidade das variáveis derivadas da curva da frequência cardíaca em teste progressivo. Lecturas: Education Física y Deportes 2005;10(90).

13. Mercier J, Legallais D, Durand M, Goudal D, Micalle JP, Préfaut C. Energy expenditure and cardiorespiratory responses at the transition between walking and running Eur J Appl Physiol Occup Physiol 1994;69(6):525-9.

14. Monteiro WD, Araujo CGS.Transição caminhada-corrida: considerações fisiológicas e perspectivas para estudos futuros. Rev Bras Med Esporte 2001;7(6):207-22.

15. Patla AE, Sparrow WA. Factors that have shaped human locomotor structure and behavior: the jewels in the crown. In: Sparrow WA, editor. Energetics of human activity. Champaign: Humans Kinetics 2000. p.43-65.

16. Carey DG, Raymond RL, Duoos BA. Intra- and inter-observer reliability in selection of the heart rate deflection point during incremental exercise: comparison to a computer generated deflection point. J Sports Sci Med 2002;1(4):115-21.

17. Kara M, Gokbel H, Bediz C, Ergene N, Üçok K, Uysal $\mathrm{H}$. Determination of the heart rate deflexion point by the Dmax method. J Sports Med Phys Fitness 1996;36(1):31-4.

18. Hughson RL, Weisiger KH, Swanson GD. Blood lactate concentration increases as continuous function in progressive exercise. J Appl Physiol 1987;62(5):1975-81.

19. Pires FO, Carmo IML, Arroyo FN, De-Oliveira FR. Característica sigmóide da FC durante teste progressivo e aplicação de diferentes métodos de identificação dos limiares de FC. Rev Mackenzie Edu Fís Esporte 2008;7(1):45-58.

20. Meyer T, Lucia A, Earnest CP, Kindermann W. A conceptual Framework for Performance Diagnosis and Training Prescription from Submaximal Gas Exchange Parameters - Theory and Application. Int J Sports Med 2005;26(suppl.1):S38-S48.

\section{Endereço para correspondência}

Eduardo Marcel Fernandes Nascimento

Rua Isabel de Castela, 478, apto 23. Vila Beatriz, CEP 05445-010 - São Paulo,SP. Brasil

E-mail: eduardomarcel@usp.br 